\title{
Procalcitonin-guided antibiotic therapy for suspected and confirmed sepsis of patients in a surgical-trauma ICU: a prospective, two-period cross-over, interventional study
}

\section{Rispah Chomba ( $\nabla$ irungurispah@yahoo.com )}

University of the Witwatersrand Faculty of Health Sciences https://orcid.org/0000-0003-3147-6688

\section{Maeyane Steve Moeng}

University of the Witwatersrand Faculty of Health Sciences

Warren Lowman

University of the Witwatersrand Faculty of Health Sciences

\section{Research article}

Keywords: Procalcitonin, trauma, sepsis, intensive care unit

Posted Date: September 27th, 2019

DOl: https://doi.org/10.21203/rs.2.15320/v1

License: (c) (1) This work is licensed under a Creative Commons Attribution 4.0 International License. Read Full License

Version of Record: A version of this preprint was published at South African Journal of Surgery on January 1st, 2020. See the published version at https://doi.org/10.17159/2078-5151/2020/v58n3a3341. 


\section{Abstract}

Background: Biomarkers like procalcitonin (PCT) are an important antimicrobial stewardship tool for critically ill patients. The purpose of our study was to compare a procalcitonin guided antibiotic algorithm to standard antibiotic treatment in surgical trauma patients admitted to the intensive care unit (ICU).

Methods: A prospective, two period cross-over study was conducted in a surgical trauma intensive care unit in South Africa. In the first period, 40 patients were recruited into the control group and antibiotics were discontinued as per standard of care. In the second period, 40 patients were recruited into the procalcitonin group and antibiotics were discontinued if the PCT decreased by $\geq 80 \%$ from the peak PCT level, or to an absolute value of less than $0.5 \mu \mathrm{g} / \mathrm{L}$. Antibiotic duration of treatment was the primary outcome. Patients were followed up for 28 days from the first sepsis event.

Results: For the first sepsis event the PCT group had a mean antibiotic duration of 9.3 days while the control group had a mean duration of 10.9 days $(p=0.10)$. Patients in the intervention group had more antibiotic free days alive (mean $7.7 \pm 6.57$ days) compared to the control group 『mean $3.8 \pm 5.22$ days, $(p=0.004 \bigotimes$. The length of ICU stay and length of hospital stay for the two groups were similar. The inhospital mortality was reduced in the intervention group (15\%) compared to the control group (30\%).

Conclusion: There was no significant difference in duration of antibiotic treatment between the two groups. However, the PCT group had more antibiotic free days alive and lower in-hospital mortality compared to the control group.

Trial registration: Pan African clinical trial registry, PACTR201909715467725, date of registration: 20.9.2019; retrospectively registered, https://pactr.samrc.ac.za/Search.aspx

\section{Introduction}

The world is facing increasing rates of multidrug resistant bacteria in intensive care units (ICUs) mainly and hospitals in general. Over-use of antibiotics is a major factor driving antibiotic resistance. There is an urgent need to implement antibiotic stewardship in ICUs to reduce antibiotic overuse. The role of biomarkers, especially procalcitonin (PCT), as antibiotic stewardship tools has been investigated in several trials(1-3). PCT levels are usually low in normal conditions but increase in severe infections and inflammatory conditions. However, the highest levels are found in septic patients. Procalcitonin rises within a few hours of infection with bacteria but reduces with appropriate antibiotic therapy (4). Procalcitonin has been used in clinical trials as a tool for monitoring clinical response to antibiotic treatment. Antibiotics are discontinued once the PCT drops to a predetermined level $[2,3,5]$. Antibiotic protocols can prevent antibiotic misuse by guiding safe discontinuation in patients who have had adequate antimicrobial therapy.

Early studies on procalcitonin guided antibiotic treatment were mostly in primary care patients with pneumonia $(5,6)$. In the ICU setting PCT has been used mainly as part of an algorithm to discontinue 
antibiotics $(1,2,7)$. Studies have proven the efficacy of PCT algorithms in reducing antibiotic duration of treatment without an increase in adverse outcomes $(8,9)$.

Studies on procalcitonin guided antibiotic therapy are mainly from Europe. There are few studies from developing countries on the use of PCT based algorithms for antibiotic stewardship. Most ICU studies contain relatively few numbers of surgical patients, and trauma patients are a unique population. It was useful to perform a study that analyses the use of established PCT algorithms specifically in surgical trauma patients in South Africa, where trauma is a major contributor to morbidity and mortality.

\section{Methods}

We conducted a prospective, two-period crossover case-controlled, interventional study in the trauma ICU at Charlotte Maxeke hospital, Johannesburg, South Africa. Eighty patients were recruited from April 2014 to July 2015. Adult patients in the trauma ICU with suspected sepsis that were either not on antibiotics or had been on antibiotic therapy for less than 48 hours were approached for consent. Patients were recruited consecutively until the sample size was achieved.

All patients in the PCT group had a serum procalcitonin done at study recruitment and then every 48 hours until antibiotics were discontinued. Procalcitonin was not used to guide antibiotic treatment in the control group.

The research protocol was approved by the Human Research Ethics Committee (HREC) of the University of the Witwatersrand, Johannesburg, South Africa.

\section{Inclusion criteria}

Patients above the age of 18 years admitted to the trauma ICU with suspected or confirmed bacterial sepsis with written consent and who survived more than 48 hours after study inclusion.

\section{Exclusion criteria}

Exclusions included patients in whom consent could not be obtained, pregnancy, patients requiring prolonged antibiotic therapy and those who had severe co-morbidities e.g. congestive cardiac failure, cirrhosis, insulin dependent diabetes, renal failure requiring dialysis, and advanced HIV infection with CD4 $\nabla 100$ cells $/ \mu \mathrm{L}$. Patients who had received more than 48 hours of antibiotics before study enrolment and those with poor chance of survival (ISS score $\geq 45$, injury critical or untreatable at screening) were also excluded.

\section{Control period}


Prior to the study antibiotics were given empirically according to the site-specific ICU algorithm and always covering at least the spectrum of previously prescribed antimicrobials as well as expected organisms. Piperacillin-tazobactam was commonly used as empiric treatment for nosocomial sepsis while amoxicillin-clavulanate was usual for community acquired infections. Antibiotics were changed to cover the spectrum of organisms cultured from the site of sepsis. The control period was between April 2014 and January 2015. Forty patients with suspected or confirmed sepsis were recruited consecutively into the control group. Antibiotics were given as per the ICU protocol above. Decisions regarding discontinuation of antibiotic treatment were left at the attending doctor's discretion.

\section{Intervention period}

In the intervention period, 40 patients with confirmed or suspected sepsis were recruited consecutively into the intervention group between February 2015 and July 2015. The PCT level was measured at study recruitment and then on alternate days. Antibiotics were given as per the ICU protocol discussed above. If the PCT decreased to an absolute value of less than $0.5 \mu \mathrm{g} / \mathrm{L}$ or by $\geq 80 \%$ from the peak PCT concentration, clinicians were encouraged to stop antibiotics. Antibiotics were not stopped if there were ongoing signs of sepsis (e.g. temperature $\geq 38.3^{\circ} \mathrm{C}$ ) with an obvious source of sepsis. The PCT complemented but did not replace clinical decision making and clinicians were able to deviate from the PCT algorithm if the need arose. Although clinicians were encouraged to stop antibiotics according to this PCT algorithm the decision to stop was at the discretion of the attending clinician.

\section{Laboratory methods}

The procalcitonin (PCT) in patient serum was measured on the ADVIA Centaur ${ }^{\circledR} B R A H M S$ PCT assay. The assay has a measurement range of $0.02-75 \mu \mathrm{g} / \mathrm{L}$ and analytical sensitivity of $0.02 \mu \mathrm{g} / \mathrm{L}$.

Microbiological specimens were processed according to standard microbiological procedures. Identification and antimicrobial susceptibility testing (AST) of cultured organisms was performed on the Vitek ${ }^{\circledR} 2$ (bioMérieux) instrument. Alternatively, AST was performed by disc diffusion or E-test ${ }^{\circledR}$ (bioMérieux). All AST results were interpreted according to the current Clinical and Laboratory Standards Institute (CLSI) criteria.

\section{Study definitions and data collection}

Further information regarding data collection and study definitions can be found in the supplementary material.

\section{Study outcomes}


The primary outcome was to determine if a procalcitonin based clinical algorithm would decrease total antibiotic days compared to standard antibiotic treatment in surgical trauma patients in an ICU setting. Secondary outcomes included antibiotic free days alive at 28 days from study inclusion, in-hospital mortality (death from any cause), ICU length of stay and recurrence or relapse of infection.

\section{Statistical analysis}

In calculating the sample size, a difference of 2 days' antibiotic duration between the mean in one group and the mean in the other group $\left(\mu_{1}-\mu_{2}\right)$ of 2 days would be significant. It was determined that 40 patients were needed in each group to detect a significant difference.

Comparisons were made between the two groups using mean ( \pm standard deviation) and t-test for continuous variables. The $p$-value was determined by a chi square but if the number of items constituting a variable was less than 10 then the $p$ value was calculated using the Fisher's exact test. Statistical significance was considered for two-sided $\mathrm{p} \otimes 0.05$. Categorical variables were compared using percentages.

Diversity between study groups was determined using chi square test and Fisher's exact test as appropriate. Risk ratio for death and infectious complications (relapse) were calculated at the $95 \%$ confidence interval. Kaplan-Meier survival curves were evaluated by the log-rank test. A multivariate analysis was not undertaken due to the small number of study subjects. Data was analysed using Statistica ${ }^{\mathrm{T}}$ version 13.2 .

\section{Results}

\section{General characteristics of the study population}

The two groups were well matched in terms of baseline demographics as shown in table 1 below. There was no significant difference in other markers of sepsis severity e.g. the SOFA score, baseline temperature, white cell count or the CRP. The PCT group however, had a worse injury severity score (ISS) than the control group and this difference was statistically significant $(p=0.002)$. Only 10 patients in the control group had a baseline PCT $(p=0.04)$.

There were 8 patients $(20 \%)$ in the control group who needed inotrope support for the first episode of sepsis compared to 5 patients $(12.5 \%)$ in the intervention group. The first sepsis event occurred approximately a day earlier in the control group (3.85 ICU days) compared to the procalcitonin group (5.05 ICU days). The main source of sepsis in both groups was pulmonary (Table 1).

\section{Microbiology culture findings}


The most commonly isolated organism in both groups of patients was ESBL-producing Klebsiella pneumoniae (Figure 1). The control group had more patients with negative cultures compared to the PCT group (6 patients vs. 2 patients). The PCT group had a slightly higher number of Pseudomonas aeruginosa infections (3 patients vs. 8 patients).

The most commonly used empiric antibiotic was piperacillin-tazobactam (PT). There were 16 patients in the control group and 36 patients in the procalcitonin group who received PT as empiric therapy. Carbapenems were more likely to be given empirically in the control group compared to the procalcitonin group.

When empiric antibiotics were assessed in terms of appropriateness to the cultured organism it was found that an equal number of patients in each group $(n=16)$ received appropriate empiric treatment (Figure 2). There were more patients in the intervention group who received inappropriate empiric antibiotics (22 patients) compared to the control group (17 patients) but the difference was not statistically significant $(p=0.59)$.

\section{Primary outcome}

When duration of therapy for the first sepsis episode was compared between groups, the procalcitonin group had a shorter duration of treatment (mean $9.3 \pm 5.67$ days) compared to the control group (10.9 \pm 2.62 days). This difference however, did not reach statistical significance $(p=0.10)$.

There were an almost equal number of patients in both groups who were treated for a second episode of sepsis (14 patients versus 15 patients). The mean duration of treatment for the second episode of sepsis was shorter in the procalcitonin group ( $9.6 \pm 2.61$ days) compared to the control group ( $12.0 \pm 4.62$ days) but the difference did not reach statistical significance $(p=0.09)$.

Clinician compliance to the procalcitonin algorithm i.e. antibiotics stopped within 24 hours of reaching the stopping criteria, was $62.5 \%$. In the non-compliant group $80 \%$ of patients had antibiotics stopped within 3 days.

\section{Secondary outcomes}

Patients in the procalcitonin group had more antibiotic free days alive (mean $7.7 \pm 6.57$ days) compared to the control group (mean $3.8 \pm 5.22$ days) $p=0.004$.

Only 2 patients (5\%) in the intervention group had a relapse of infection compared to 9 patients $(22.5 \%)$ in the control group $(p=0.02)$.

There was no difference in length of ICU stay between the procalcitonin group (mean $16.1 \pm 8.31$ days) compared to the control group (mean $17.6 \pm 13.84$ days), $p=0.5$. The procalcitonin group had a slightly 
longer hospital stay ( $25.4 \pm 8.32$ days) than the control group ( $24.5 \pm 17.03$ days) but the difference was not statistically significant $(p=0.76)$.

The PCT group had fewer deaths (6 patients, 15\%) compared to the control group (12 patients, 30\%). Survival between the two groups was compared using Kaplan-Meier analysis as shown in figure 3 below. Complete cases were patients who suffered a death event. Censored cases were patients alive who did not suffer the event (death). The intervention group had improved survival compared to the control group (Wilcoxon log rank analysis had a $p$ value of 0.045 ).

\section{Discussion}

In this single centre study, a procalcitonin-based algorithm failed to demonstrate a reduction in duration of antibiotic treatment in surgical trauma patients. The procalcitonin group had shorter duration of treatment for both the first and second episode of sepsis compared to the control group but this did not translate into a statistically significant difference. This is in contrast to other studies which found that use of a PCT-based antibiotic algorithm reduces duration of antibiotics in critically ill septic patients $(2,7,10)$. This difference is possibly due to poor clinician compliance with a procalcitonin algorithm.

However, an important finding of this study was a significant increase in antibiotic free days alive at 28 days in the procalcitonin group compared to controls. Other researchers have reported similar findings. In one such study 101 patients were randomized to either a procalcitonin group or a control group. The PCT group had more antibiotic-free days alive (13 days) compared to the control group (9.5 days) (11). In one systematic review of six ICU studies there was a $23-37 \%$ increase in antibiotic free days alive in the PCT group compared to the control group(12). Our results show that the procalcitonin group had lower inhospital mortality compared to controls. Similarly, other authors report reduced mortality in the PCT group compared to the control group $(1,3,13)$. The reduction in mortality and increased antibiotic free days alive may relate to improved management of septic episodes. Use of a PCT algorithm facilitates decisions around antimicrobial therapy, whereby initiation of treatment and response to treatment are guided not only clinically but also by an objective laboratory marker.

Our study found a small but insignificant reduction in ICU stay in the procalcitonin group. The effects of a PCT-based algorithm on length of ICU stay are not very clear when compared across different studies. One study found a significantly shortened ICU stay in the PCT group compared to the control group (7). Another study however, found that length of ICU stay was one day longer in patients in the PCT arm compared to the standard-of-care arm (14). This study differs from other studies because it used an algorithm of PCT-guided antibiotic escalation which prolonged ICU admission without improving survival. A meta-analysis of seven studies comprising 1,075 patients with septic shock or severe sepsis compared PCT-guided antibiotic treatment to standard of care (15). It reported no appreciable difference the ICU length of stay between the PCT and the control groups. The effect of a PCT-based antibiotic algorithm on length of ICU stay is therefore still open to debate. 
Our results showed no difference in hospital stay between the procalcitonin and control groups. Other authors $(1,3,15,16)$ have similarly found no difference in duration of hospital stay when comparing procalcitonin guided strategies to standard of care.

A major concern whilst using a PCT algorithm to discontinue antibiotics is the potential for relapse if infections are inadequately treated. Our study had a lower relapse rate in the procalcitonin group compared to the control group. Patients in the PCT group may have benefited from a more focused assessment and vigorous source control leading to the lower relapse rate, which again highlights the potential of a PCT algorithm in management of septic. A meta-analysis of 14 trials where patients were assigned to receive antibiotics based on a PCT algorithm similarly found decreased risk of treatment failure in patients assigned to a PCT group (17).

Some authors however, have found no difference in relapse rates when comparing PCT group to controls (7). One study however, found that re-institution of antibiotics for relapse was more common in the PCT group than in the control group but the numbers were small in both instances (5\% versus $3 \%)(3)$.

To our knowledge, this is the first prospective study evaluating the value of a PCT-based algorithm in reducing antibiotic duration of treatment in surgical trauma ICU patients.

Our study had several limitations; firstly, it was a single centre, non-randomized study. The results of this study may be biased by non-randomization and may not be extrapolated to patients with a different background. However, since this was a single centre study a crossover design was best suited to the study aims. Secondly, the sample size was calculated to have sufficient power to detect a between group difference of at least two days in antibiotic duration of treatment. It is likely that a larger sample size would have detected a statistically significant difference between groups with the results that we obtained. The small sample also precluded a multivariate analysis. Thirdly, poor compliance by clinicians to a PCT algorithm may have resulted in a conservative bias and reduced the potential benefits of a PCTguided algorithm. Fourthly, our definition of relapse was defined using microbiologic criteria. Patients who were discharged home or transferred out may have suffered a late relapse that was underestimated in our study. However, the reduced relapse rate in the procalcitonin group supports the safety of PCT based antibiotic algorithms. Finally, our study did not measure the actual reduction in antibiotic costs associated with a PCT algorithm. Other studies have reported a decline in antibiotic costs using PCT algorithms, without any associated adverse outcomes (18)

\section{Conclusion}

Our study showed that procalcitonin has the potential to reduce the duration of antibiotics for surgical trauma patients with suspected and confirmed sepsis. Although not reaching statistical significance, the PCT group had reduced duration of antibiotic treatment for both the first and the second episode of sepsis. We observed a significant increase in antibiotic free days alive, improved survival and a lower relapse rate for patients managed according to a PCT algorithm. This reduction in antibiotic usage has the added potential benefit of reducing antibiotic costs. Procalcitonin can be measured on alternate days 
or longer intervals in resource limited settings to reduce costs while still retaining the observed benefits. The potential of procalcitonin guided regimens to reduce mortality and increase survival is difficult to quantify in terms of cost benefit and needs to be explored in further studies.

\section{List Of Abbreviations}

AST - antibiotic susceptibility testing

CLSI - Clinical and Laboratory Standards Institute

CPAP - continuous positive airway pressure

CRP - C-reactive protein

ESBL - extended spectrum beta lactamase producer

GCS - Glasgow coma scale

HIV - human immunodeficiency virus

ICU - intensive care unit

ISS - injury severity score

PCT - procalcitonin

PT - piperacillin-tazobactam

SIMV - synchronized intermittent mandatory ventilation

SOFA - sequential organ failure assessment

SSTI - skin and soft tissue infection

UTI - urinary tract infection

\section{Declarations}

\section{Ethics approval and consent to participate}

The research protocol was approved by the Human Research Ethics Committee (HREC) of the University of the Witwatersrand. The ethics clearance certificate number is M130860. All study participants had to give written informed consent to participate in the study. Relatives were approached for written informed consent for patients who could not consent. 


\section{Consent for publication}

Not applicable

\section{Availability of data and materials}

Data generated during the current study is available from the corresponding author on reasonable request.

\section{Competing interests}

The authors declare that they have no competing interests.

\section{Funding}

Not applicable

\section{Authors, contributions}

Conceived and designed the study: WL, RC. Performed the study: RC, SM. Analysed the data: RC, WL. Wrote the paper: RC, SM, WL. All authors drafted the manuscript, revised it and approved the final manuscript.

\section{Acknowledgements}

Not applicable

\section{References}

1. Bouadma L, Luyt C-E, Tubach F, Cracco C, Alvarez A, Schwebel C, et al. Use of procalcitonin to reduce patients' exposure to antibiotics in intensive care units (PRORATA trial): a multicentre randomised controlled trial. Lancet. 2010 Feb 6;375(9713):463-74.

2. Hochreiter M, Köhler T, Schweiger AM, Keck FS, Bein B, von Spiegel T, et al. Procalcitonin to guide duration of antibiotic therapy in intensive care patients: a randomized prospective controlled trial. Crit Care. 2009;13(3): R83.

3. de Jong E, van Oers JA, Beishuizen A, Vos P, Vermeijden WJ, Haas LE, et al. Efficacy and safety of procalcitonin guidance in reducing the duration of antibiotic treatment in critically ill patients: a 
randomised, controlled, open-label trial. Lancet Infect Dis. 2016 Jul;16(7):819-27.

4. Becker KL, Snider R, Nylen ES. Procalcitonin in sepsis and systemic inflammation: a harmful biomarker and a therapeutic target. Br J Pharmacol. 2010 Jan 1;159(2):253-64.

5. Christ-Crain M, Jaccard-Stolz D, Bingisser R, Gencay MM, Huber PR, Tamm M, et al. Effect of procalcitonin-guided treatment on antibiotic use and outcome in lower respiratory tract infections: cluster-randomised, single-blinded intervention trial. Lancet. 2004 Feb 21;363(9409):600-7.

6. Briel M, Schuetz P, Mueller B, Young J, Schild U, Nusbaumer C, et al. Procalcitonin-guided antibiotic use vs a standard approach for acute respiratory tract infections in primary care. Arch Intern Med. 2008 Oct 13;168(18):2000-7; discussion 2007-2008.

7. Nobre V, Harbarth S, Graf J-D, Rohner P, Pugin J. Use of procalcitonin to shorten antibiotic treatment duration in septic patients: a randomized trial. Am J Respir Crit Care Med. 2008 Mar 1;177(5):498505.

8. Broyles MR. Impact of Procalcitonin-Guided Antibiotic Management on Antibiotic Exposure and Outcomes: Real-world Evidence. Open Forum Infect Dis. 2017;4(4):ofx213.

9. Wirz Y, Meier MA, Bouadma L, Luyt CE, Wolff M, Chastre J, et al. Effect of procalcitonin-guided antibiotic treatment on clinical outcomes in intensive care unit patients with infection and sepsis patients: a patient-level meta-analysis of randomized trials. Crit Care. 2018 Aug 15;22(1):191.

10. Albrich WC, Dusemund F, Bucher B, Meyer S, Thomann R, Kühn F, et al. Effectiveness and safety of procalcitonin-guided antibiotic therapy in lower respiratory tract infections in "real life": an international, multicenter poststudy survey (ProREAL). Arch Intern Med. 2012 May 14;172(9):715-22.

11. Stolz D, Smyrnios N, Eggimann P, Pargger H, Thakkar N, Siegemund M, et al. Procalcitonin for reduced antibiotic exposure in ventilator-associated pneumonia: a randomised study. Eur Respir J. 2009 Dec;34(6):1364-75.

12. Agarwal R, Schwartz DN. Procalcitonin to guide duration of antimicrobial therapy in intensive care units: a systematic review. Clin Infect Dis. 2011 Aug;53(4):379-87.

13. Schuetz P, Wirz Y, Sager R, Christ-Crain M, Stolz D, Tamm M, et al. Effect of procalcitonin-guided antibiotic treatment on mortality in acute respiratory infections: a patient level meta-analysis. Lancet Infect Dis. 2018 Jan;18(1):95-107.

14. Jensen JU, Hein L, Lundgren B, Bestle MH, Mohr TT, Andersen MH, et al. Procalcitonin-guided interventions against infections to increase early appropriate antibiotics and improve survival in the intensive care unit: a randomized trial. Crit Care Med. 2011 Sep;39(9):2048-58.

15. Prkno A, Wacker C, Brunkhorst FM, Schlattmann P. Procalcitonin-guided therapy in intensive care unit patients with severe sepsis and septic shock-a systematic review and meta-analysis. Crit Care. 2013 Dec 11;17(6): R291.

16. Lam SW, Bauer SR, Fowler R, Duggal A. Systematic Review and Meta-Analysis of ProcalcitoninGuidance Versus Usual Care for Antimicrobial Management in Critically III Patients: Focus on Subgroups Based on Antibiotic Initiation, Cessation, or Mixed Strategies. Crit Care Med. 2018 May;46(5):684-90. 
17. Schuetz P, Briel M, Christ-Crain M, Stolz D, Bouadma L, Wolff M, et al. Procalcitonin to guide initiation and duration of antibiotic treatment in acute respiratory infections: an individual patient data metaanalysis. Clin Infect Dis. 2012 Sep;55(5):651-62.

18. Schroeder S, Hochreiter M, Koehler T, Schweiger A-M, Bein B, Keck FS, et al. Procalcitonin (PCT)guided algorithm reduces length of antibiotic treatment in surgical intensive care patients with severe sepsis: results of a prospective randomized study. Langenbecks Arch Surg. 2009 Mar;394(2):221-6.

\section{Tables}

Table 1: General characteristics of the study population 


\begin{tabular}{|c|c|c|c|}
\hline Characteristic & $\begin{array}{l}\text { Control group } \\
(n=40)\end{array}$ & $\begin{array}{l}\text { PCT group } \\
(n=40)\end{array}$ & $\begin{array}{l}\mathrm{p} \\
\text { value }\end{array}$ \\
\hline Age in years (mean $\pm S D$ ) & $36.1 \pm 14.7$ & $36.0 \pm 12.1$ & 0.96 \\
\hline \multicolumn{4}{|l|}{ Gender, n (\%) } \\
\hline Male & 39 (97.5\%) & $33(82.5 \%)$ & \\
\hline Female & $1(2.5 \%)$ & $7(17.5 \%)$ & \\
\hline \multicolumn{4}{|l|}{ Mechanical ventilation type, $n$ (\%) } \\
\hline $\begin{array}{l}\text { Synchronized intermittent mechanical ventilation } \\
\text { (SIMV) }\end{array}$ & $26(66.7 \%)$ & $27(69.2 \%)$ & - \\
\hline Continuous positive airway pressure (CPAP) & $13(33.3 \%)$ & $12(30.8 \%)$ & - \\
\hline SOFA score (mean $\pm \mathrm{SD}$ ) & $7.3 \pm 2.92$ & $7.1 \pm 2.98$ & 0.70 \\
\hline ISS score (mean \pm SD) & $20.3 \pm 10.18$ & $26.8 \pm 8.17$ & 0.002 \\
\hline GCS score (mean \pm SD) & $7.1 \pm 3.68$ & $6.1 \pm 3.02$ & 0.21 \\
\hline \multicolumn{4}{|l|}{ Sepsis markers (mean $\pm \mathrm{SD}$ ) } \\
\hline Temperature ${ }^{\circ} \mathrm{C}$ & $37.5 \pm 0.89$ & $37.1 \pm 5.94$ & 0.70 \\
\hline Total white cell count $\left(\mathrm{x} 10^{9} / \mathrm{L}\right)$ & $14.5 \pm 8.92$ & $15.4 \pm 6.45$ & 0.6 \\
\hline $\mathrm{CRP}(\mathrm{mg} / \mathrm{L})$ & $227.8 \pm 119.89$ & $263.1 \pm 91.26$ & 0.14 \\
\hline PCT $(\mu \mathrm{g} / \mathrm{L})$ & $\begin{array}{l}91.0 \pm 159.62 \\
(\mathrm{n}=10)\end{array}$ & $\begin{array}{l}29.2 \pm 58.13(n= \\
40)\end{array}$ & 0.04 \\
\hline \multicolumn{4}{|l|}{ Co-morbid illnesses, n (\%) } \\
\hline None & $34(85 \%)$ & $31(77 \%)$ & - \\
\hline Cardiac & $2(5 \%)$ & $1(2.5 \%)$ & - \\
\hline
\end{tabular}




\begin{tabular}{|c|c|c|c|}
\hline Neurologic & $2(5 \%)$ & $1(2.5 \%)$ & - \\
\hline HIV & $1(2.5 \%)$ & $6(15 \%)$ & - \\
\hline Chronic lung & $1(1 \%)$ & 0 & - \\
\hline Endocrine & 0 & $1(2.5 \%)$ & - \\
\hline \multicolumn{4}{|c|}{ Received antibiotics prior to study inclusion, $\mathrm{n}$ (\%) } \\
\hline Surgical prophylaxis & 5 & 2 & - \\
\hline Sepsis & 4 & 2 & - \\
\hline \multicolumn{4}{|l|}{ Reason for ICU admission, $\mathrm{n}(\%)$} \\
\hline Mechanical ventilation & $22(55 \%)$ & $15(37.5 \%)$ & - \\
\hline Haemodynamic instability & $5(12.5 \%)$ & $3(7.5 \%)$ & - \\
\hline Major surgery & $3(7.5 \%)$ & $1(2.5 \%)$ & - \\
\hline Severe injury & $8(20 \%)$ & $21(52.5 \%)$ & - \\
\hline Sepsis & $2(5 \%)$ & 0 & - \\
\hline \multicolumn{4}{|l|}{ Source of first episode sepsis, $n$ (\%) } \\
\hline Pulmonary & $16(40 \%)$ & $18(45 \%)$ & - \\
\hline SSTIs & $9(22.5 \%)$ & $5(12.5 \%)$ & - \\
\hline Abdominal & $8(20 \%)$ & $3(7.5 \%)$ & - \\
\hline Urinary tract infection (UTI) & $1(2.5 \%)$ & $4(10 \%)$ & - \\
\hline Primary Bloodstream & $3(7.5 \%)$ & $8(20 \%)$ & - \\
\hline Catheter related blood stream infection & 0 & $2(5 \%)$ & - \\
\hline Unknown & $3(7.5 \%)$ & 0 & - \\
\hline
\end{tabular}


Figures

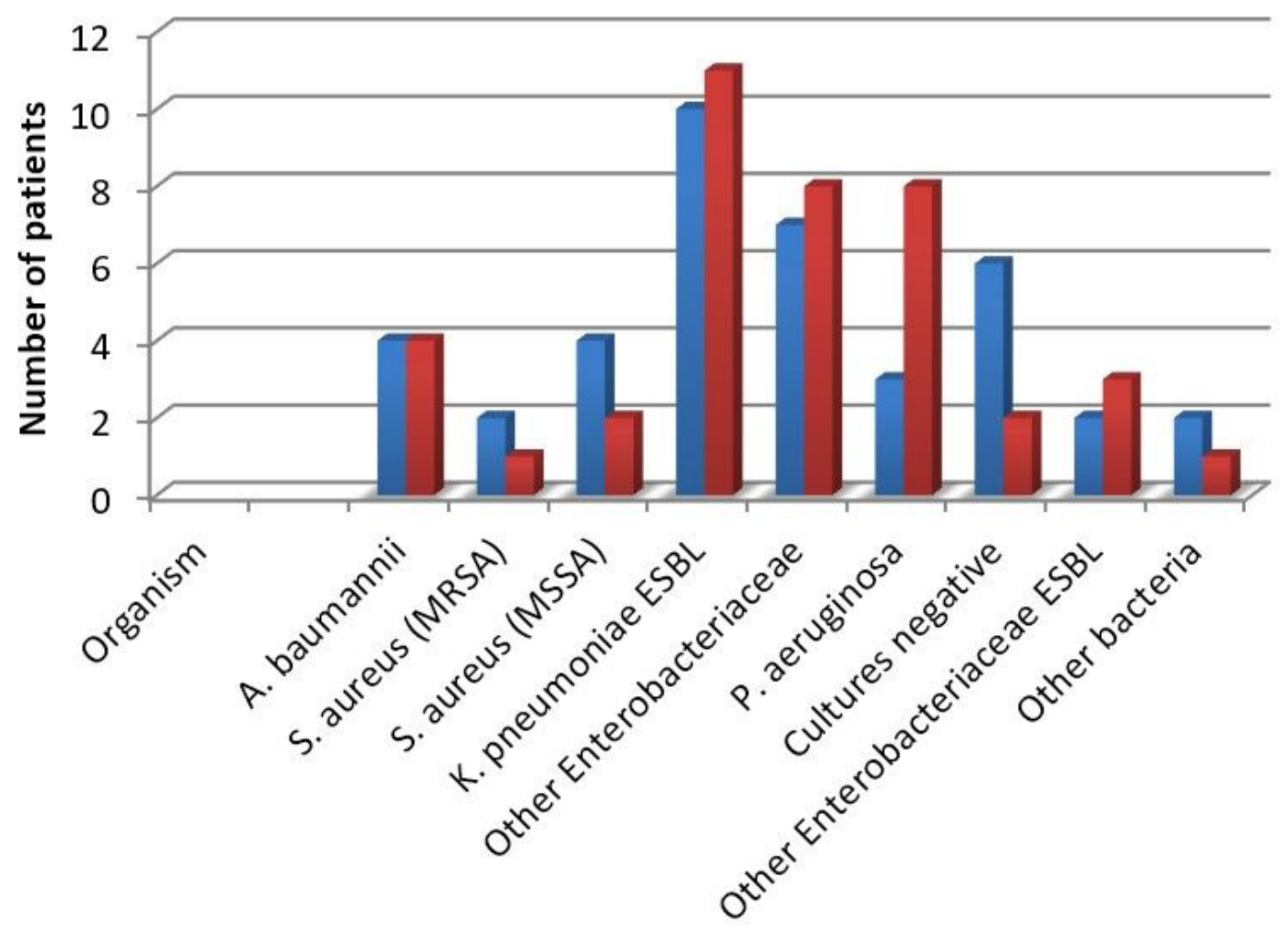

Control group

- Procalcitonin group

\section{Primary isolate from cultures}

Figure 1

Primary bacterial isolates from culture 


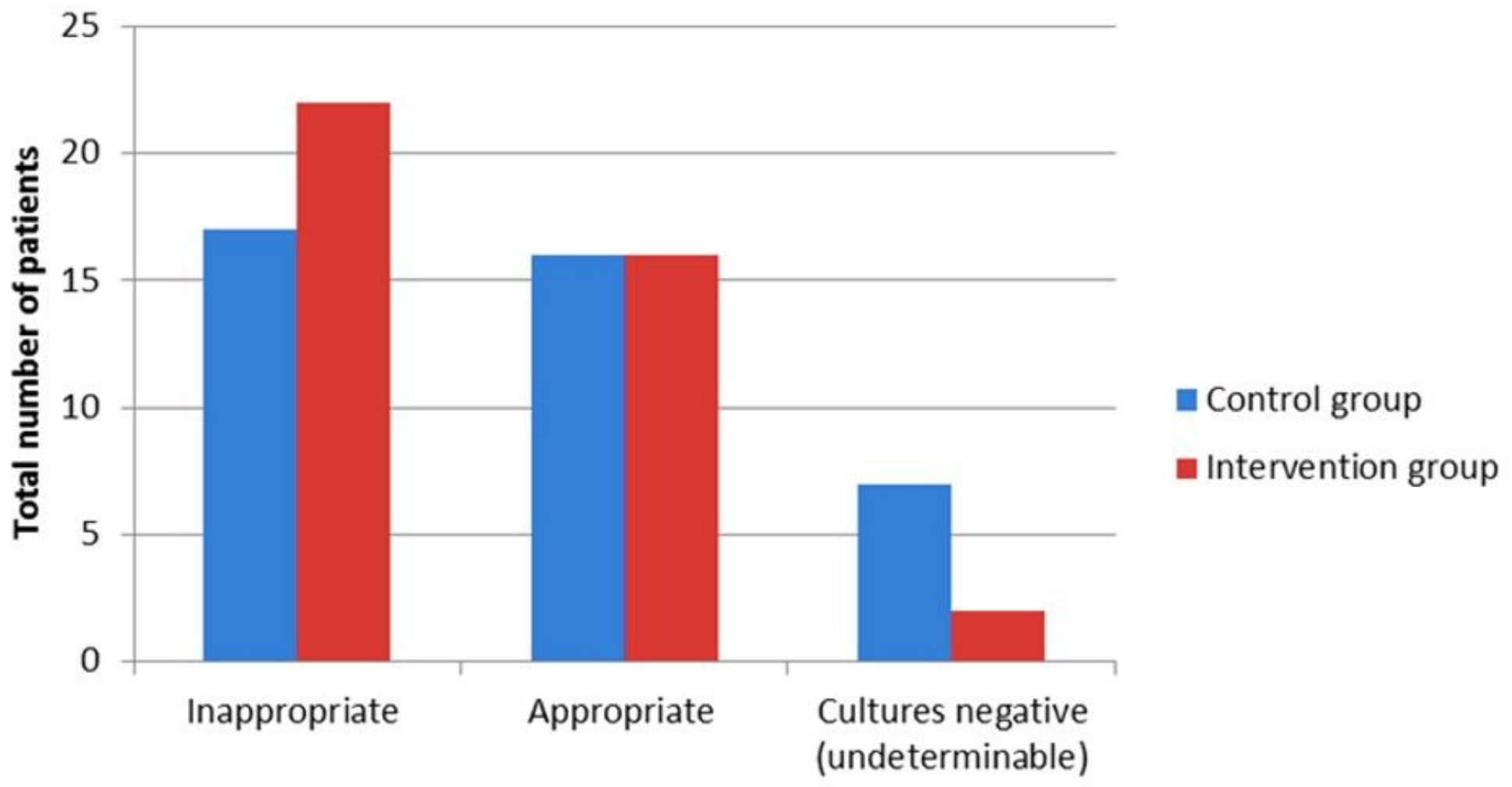

Appropriateness of empiric antibiotics

Figure 2

Appropriateness of empiric antibiotic treatment 


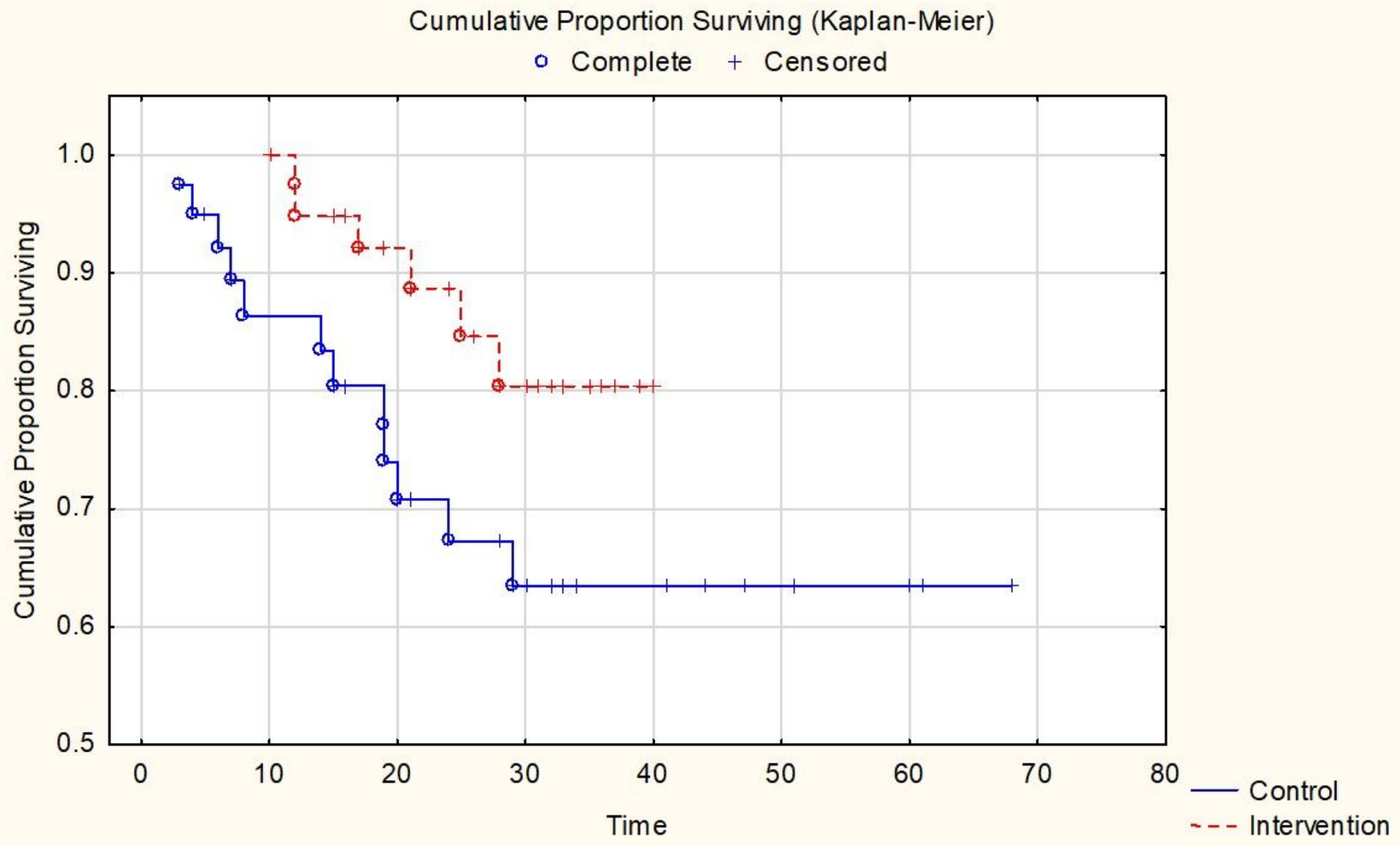

Figure 3

Kaplan-Meier survival curve

\section{Supplementary Files}

This is a list of supplementary files associated with this preprint. Click to download.

- Empiricantibioticsforfirstsepsisevent.docx

- Datacollectionsheet.docx

- Definitionsofterminologiesusedinthestudy.docx 